\title{
Modelagem matemática do comportamento térmico de subestações subterrâneas considerando dados reais
}

\section{Mathematical modeling of the thermal behavior of underground substations considering real data}

\author{
Taciana Paula Enderle ${ }^{(}, 1$, Airam Tereza Zago Sausen ${ }^{(}, 1$, Maurício de \\ Campos ${ }^{(0)}$, Paulo Sérgio Sausen ${ }^{(\bullet, 1}$
}

${ }^{1}$ Programa de Pós-Graduação Stricto Sensu em Modelagem Matemática (PPGMM), UNIJUÍ, Ijuí (RS) - Brasil

*taciana.enderle@unijui.edu.br; airam@unijui.edu.br; campos@unijui.edu.br; sausen@unijui.edu.br

Recebido: 15/10/2019. Revisado: 05/06/2020. Aceito: 20/07/2020.

\section{Resumo}

Os transformadores têm um papel fundamental nos sistemas de distribuição de energia, e com a evolução das redes elétricas para redes inteligentes a instrumentação destes equipamentos torna-se necessária. Modelos matemáticos que representam corretamente o comportamento dos transformadores e de suas subestações são fundamentais, uma vez que poderão compor novas configurações, além de permitir análises futuras de operação. Nesse contexto, neste artigo é realizada a modelagem matemática do comportamento térmico de uma subestação subterrânea do sistema de distribuição de uma concessionária de energia da região sul do Brasil. São utilizados dados reais de operação obtidos através de um sistema de monitoramento remoto de uma rede de distribuição de energia subterrânea por um período de 72 horas abrangendo duas estações do ano, inverno e verão. A validação do modelo ocorre a partir da comparação entre as temperaturas presentes na subestação e as calculadas pelo modelo. A partir dos resultados das simulações é possível verificar que para ambas estações do ano o modelo apresentou erros médios inferiores a 3,1\%, dentro do que é esperado para modelos térmicos segundo a literatura técnica.

Palavras-Chave: Modelagem Matemática; Temperatura; Transformadores; Redes Subterrâneas.

\section{Abstract}

Transformers have a fundamental role in energy distribution systems, and with the evolution of electrical networks to intelligent networks, the instrumentation of this equipment is a necessity. Mathematical models that correctly represent the behavior of transformers and their substations are essential, since they will be able to compose new configurations, in addition to allowing future analysis of operation. In this context, this paper performs the mathematical modeling of the thermal behavior of an underground substation of the distribution system of an energy concessionaire in southern Brazil. Actual operating data are used, they are obtained through a remote monitoring system of an underground energy distribution network for a period of 72 hours, covering two seasons, winter and summer. The model validation occurs through comparative analysis between the temperature in the substation and those calculated by the model. From simulations results, it is possible to verify that, for the two seasons, the model presented average errors below 3,1\%, within the expected for thermal models according to the technical literature.

Keywords: Mathematical Modeling; Temperature; Transformers; Underground Networks. 


\section{Introdução}

A partir dos primeiros registros da utilização da energia elétrica no Brasil, em meados de 1880 , seu uso tornouse indispensável, devido ao atual modo de vida da população, a sociedade industrializada, e ao crescente desenvolvimento tecnológico mundial. Desde então, a busca por um fornecimento confiável e de qualidade deste insumo, vem orientando as diretrizes operacionais das concessionárias de distribuição de energia elétrica. Além disso, este tema tem se tornado cada vez mais objeto de estudo e pesquisa, e também alvo de fiscalização do setor elétrico, em função das exigências legais, dos avanços tecnológicos e do próprio crescimento populacional.

Neste sentido, o Sistema Elétrico de Potência (SEP) é o responsável por fornecer energia elétrica atendendo a quase todos os consumidores. Conforme Theis (2008), o SEP pode ser classificado em quatro diferentes áreas: sistemas de geração, redes de transmissão, redes de distribuição, e consumidores (cargas). Ainda, as linhas de transmissão e distribuição são classificadas conforme o nível de tensão: as tensões de distribuição são tipicamente de 10 a $60 \mathrm{kV}$, as tensões de subtransmissão são de 66 a $138 \mathrm{kV}$ e as tensões de transmissão estão normalmente acima de $138 \mathrm{kV}$. Além disso, as redes de distribuição de energia elétrica podem ser classificadas de várias formas, resultando em diversas combinações entre redes de baixa e média tensão.

Dentre estas classificações, as redes de distribuição podem ser divididas em dois tipos: redes aéreas e redes subterrâneas. Sendo que em ambas as instalações, baseado nos conceitos atuais, o equipamento utilizado para condicionar os níveis de tensão em diversos pontos do circuito é o transformador. Segundo Shirvani et al. (2010), o transformador de potência é um elemento importante do sistema, tanto de transmissão, como de distribuição de energia elétrica. Existe um conjunto de fatores que interfere no seu funcionamento/comportamento, tais como: frequência, temperatura, fator de carga, características construtivas, e até mesmo a regularidade das suas manutenções.

Como todo equipamento, o transformador está sujeito a falhas, neste sentido, Gouda et al. (2013) divide essas falhas em duas classes: internas e externas. Considerando as falhas internas, essas atingem entre 70 e $80 \%$ das falhas apontadas nos transformadores quando submetidos a uma observação de 20 anos. Este tipo de falha ocorre de forma inesperada e geralmente requer uma ação rápida pelo relé de proteção para desconectar o transformador do sistema de energia elétrica.

Do ponto de vista financeiro, conforme Rigatos et al. (2012) e Zhou et al. (2014), os transformadores de potência estão entre os elementos que possuem maior custo financeiro no sistema de transmissão e distribuição de energia elétrica. Desta forma, o monitoramento das condições de operação dos mesmos é um fator importante para o funcionamento ininterrupto e confiável da rede elétrica. Além disso, durante a sua vida útil, os transformadores normalmente exibem um alto nível de confiabilidade em condições normais de funcionamento, a partir de um nível adequado de manutenção.
Lockie and Whirlow (1968), em um dos primeiros trabalhos sobre redes subterrâneas, destacam que os transformadores neste tipo de rede são expostos a um ambiente diferente dos transformadores instalados em redes aéreas. Estas diferenças podem afetar sua capacidade de carga, uma vez que sua refrigeração é diretamente prejudicada pelas condições de instalação. Por sua vez, a vida útil do transformador está diretamente associada ao carregamento ao qual o mesmo está submetido. Porém, seu desgaste tende a aumentar em decorrência dos efeitos térmicos e variações de tensão.

Nem sempre é possível testar este equipamento para todas as condições as quais ele é submetido ao longo da sua vida útil. Então, a modelagem matemática do comportamento térmico da subestação passa a ser um fator importante, uma vez que permite a simulação do seu comportamento ou ainda, pode permitir análises que interferem diretamente na tomada de decisões futuras. Deste modo, é desejável que o modelo representativo do sistema seja o mais acurado possível.

Neste contexto, neste artigo é apresentada a modelagem matemática do comportamento térmico de uma subestação subterrânea do sistema de distribuição de energia, que pertence a Companhia Estadual de Energia Elétrica do Rio Grande do Sul (CEEE/RS). As simulações são realizadas a partir do uso do software Matlab e os resultados obtidos, pelo modelo proposto, são comparados com dados reais de uma subestação subterrânea de energia e de seu respectivo transformador.

O trabalho está organizado como segue. Na Seção 2 são apresentados os trabalhos relacionados à temática desta pesquisa através de uma revisão bibliográfica da literatura técnica. Na Seção 3 é detalhado o sistema de distribuição subterrâneo utilizado na pesquisa. Na Seção 4 é apresentado o modelo térmico da subestação, composto de seu circuito equivalente e das suas equações. Na Seção 5 são apresentados os resultados das simulações e a validação do modelo proposto. Por fim, na Seção 6 são apresentadas as conclusões e as possibilidades de trabalhos futuros.

\section{Revisão Bibliográfica}

Um número significativo de modelos matemáticos de transformadores pode ser encontrado na literatura, relacionando os diversos fatores que podem interferir na operação do transformador, e os efeitos provocados em cada um dos casos. Dentre estes modelos, este artigo priorizou aqueles que estão associados as implicações das variações de temperatura, uma vez que esta variável tem efeito direto na vida útil afetando as características construtivas destes dispositivos.

A instalação de transformadores de distribuição em câmaras subterrâneas impõe requisitos de refrigeração mais rigorosos do que a montagem em postes. Um dos primeiros trabalhos que propôs um modelo matemático para prever o comportamento térmico de subestações de distribuição subterrânea, sob vários tipos de carga, foi desenvolvido por Whirlow and Lockie (1968). Este modelo é um modelo clássico bastante referenciado em estudos de redes de distribuição subterrâneas 
quando o tema envolve análise térmica, e a partir dele diversos outros trabalhos surgiram na literatura (Djamali and Tenbohlen, 2017, Djamali et al., 2018, Sandraz et al., 2013). O modelo de Whirlow and Lockie (1968) considera que os componentes, tanto internos quanto externos possuem uma capacitância térmica e uma resistência térmica em relação ao fluxo de calor, de componente para componente. A temperatura de cada um deles é considerada a partir de uma média da variação parcial da temperatura do componente. $\mathrm{Na}$ Fig. 1 pode-se observar o modelo da configuração física do transformador, e na Fig. 2 o seu circuito térmico equivalente.

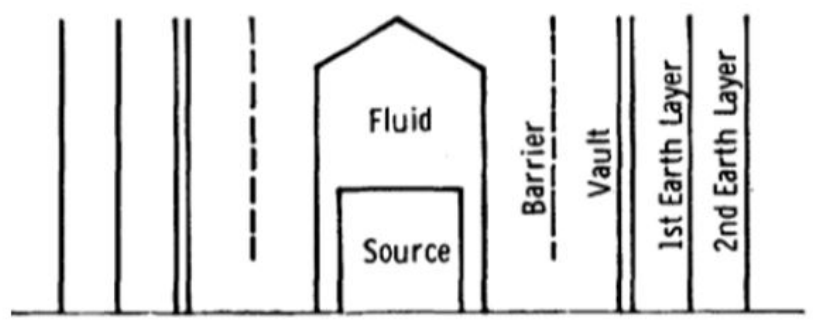

Figura 1: Configuração física do modelo.

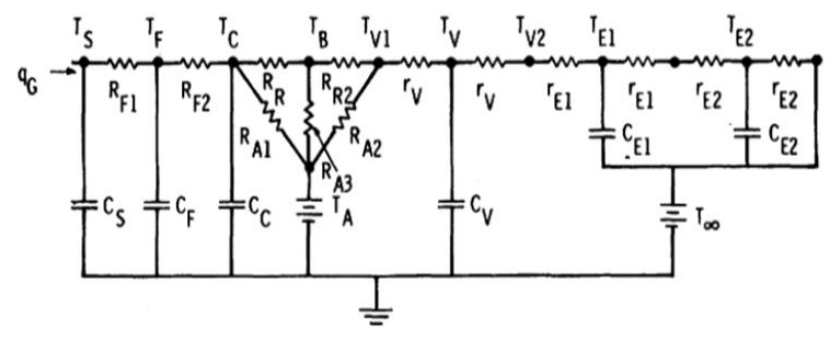

Figura 2: Circuito térmico do modelo.

A capacitância térmica, na Eq. (1), é dada por:

$$
C=\rho C_{p} v
$$

sendo que: $\rho$ é a densidade $(\mathrm{g} / \mathrm{ml}), C_{p}$ é o calor específico $(\mathrm{cal} /(\mathrm{g} . \mathrm{C}))$, e $v$ é o volume $(\mathrm{ml})$.

A resistência térmica para a transferência de calor condutora, na Eq. (2), é encontrada pela lei de Fourier:

$$
q=-k A \frac{\Delta T}{\Delta x}
$$

sendo que: $k$ é a condutividade térmica $\left(W /\left(m^{2} . C\right)\right), A$ é a área $\left(m^{2}\right), \Delta T$ é a variação de temperatura $(C)$ e $\Delta x$ é a variação da distância $(m)$.

No trabalho desenvolvido por Gouvea et al. (2004) é apresentado o detalhamento de um sistema de equações diferenciais que rege a troca de calor em um transformador de potência em uma câmara subterrânea, para o estabelecimento da temperatura do ponto quente do seu enrolamento. $\mathrm{O}$ carregamento admissível para a operação em condições normais e de primeira contingência é determinado a partir do estabelecimento da perda de vida útil em função do tipo de transformador e das características de ventilação da câmara subterrânea. São consideradas duas metodologias de estudo, na primeira, a temperatura interna da câmara é determinada por meio do balanço térmico resultante entre o fluxo de calor produzido pelas perdas elétricas no transformador, a temperatura ambiente do meio externo e a transferência de calor para o meio externo, pelas paredes, pelo teto e pela ventilação forçada. $\mathrm{O}$ equacionamento interno é realizado pelas Eqs. (3) a (6), considerando que o transformador está instalado ao ar livre, exceto pelo fato de que a temperatura interna da câmara é calculada pela transferência de calor produzida no transformador pelas paredes e teto da câmara, ou seja:

$$
Q_{\text {TRAFO }}=Q_{\text {PAREDES }}+Q_{\text {TETO }}+Q_{V E N T}
$$

sendo que:

$$
Q_{\text {PAREDES }}=0,7 \times h_{\text {PAREDES }} \times A_{\text {PAREDES }}\left(T_{C}-T_{\text {SOLO }}\right)
$$

$$
\begin{gathered}
Q_{\text {TETO }}=h_{\text {TETO }} \times A_{\text {TETO }}\left(T_{C}-T_{A M B}\right) \\
Q_{V E N T}=m_{A R} \times C_{A R}\left(T_{C}+T_{A M B}\right)
\end{gathered}
$$

sendo que: $Q_{\text {TRAFO }}$ é a quantidade de calor produzida pelas perdas no transformador $(W), Q_{\text {PAREDES }}$ é a quantidade de calor dispersa para o solo através das paredes da câmara $(W), Q_{\text {TETO }}$ é a quantidade de calor dispersa para o meio através do teto da câmara $(W), Q_{V E N T}$ é a quantidade de calor extraída da câmara pela ventilação forçada $(W), h_{\text {PAREDES }}$ é o coeficiente de transferência de calor pela parede $\left(W /\left(m^{2} . K\right)\right), h_{\text {TETO }}$ é o coeficiente de transferência de calor pelo teto $\left(W /\left(m^{2} . K\right)\right), A_{P A R E D E S}$ é a área total da superfície da parede $\left(m^{2}\right), A_{\text {TETO }}$ é a área total da superfície do teto $\left(m^{2}\right), m_{A R}$ é a vazão mássica do ar $(\mathrm{kg} / \mathrm{s}), C_{A R}$ é a capacidade térmica do $\operatorname{ar}(\mathrm{J} /(\mathrm{kg} . \mathrm{K}))$, $T_{C}$ é a temperatura média da câmara $\left({ }^{\circ} \mathrm{C}\right), \mathrm{T}_{S O L O}$ é a temperatura média do solo $\left({ }^{\circ} \mathrm{C}\right)$ e $T_{A M B}$ é a temperatura ambiente, externa à câmara $\left({ }^{\circ} \mathrm{C}\right)$,

Na segunda metodologia, a transferência de calor produzida pelo transformador, pelas perdas no enrolamento e no núcleo de ferro, é regida pela equação diferencial Eq. (7), dada por:

$$
C_{\text {TRAFO }} \frac{d T_{\text {TRAFO }}}{d t}=Q_{\text {TRAFO }}(t)-Q_{P}(t)
$$

sendo que: $T_{T R A F O}$ é a temperatura do transformador $\left({ }^{\circ} \mathrm{C}\right), C_{\text {TRAFO }}$ é a capacidade térmica do transformador $\left(W h /{ }^{\circ} \mathrm{C}\right), Q_{T R A F O}(t)$ é a quantidade de calor produzida pelas perdas no transformador $(W)$, e $Q_{P}(t)$ é o fluxo de calor transferido ao ar e as paredes da câmara $(W)$.

A transferência de calor do transformador para o ar e para as paredes da câmara é realizada por convecção 
e por radiação térmica. Na Eq. (8) é apresentada a transferência de calor do transformador para a câmara:

$$
Q_{P}=Q_{C O N V}+Q_{R A D}
$$

nas Eqs. (9) e (10) são expressos $Q_{C O N V}$ e $Q_{R A D}$ :

$$
\begin{gathered}
Q_{C O N V}=h_{\text {TRAFO }} A_{\text {TRAFO }}\left(T_{\text {TRAFO }}-T_{A R}\right) \\
Q_{R A D}=\frac{\sigma A_{\text {TRAFO }}\left(A_{\text {TRAFO }}^{4}-T_{A R}^{4}\right)}{R_{T}}
\end{gathered}
$$

e $R_{T}$ é dado pela Eq. (11):

$$
R_{T}=\frac{1}{\varepsilon_{T R A F O}}+\frac{1-\varepsilon_{C}}{\varepsilon_{C}} \frac{A_{T R A F O}}{A_{C}}
$$

sendo que: $Q_{C O N V}$ é o calor transferido ao ar no interior da câmara por convecção $(W), Q_{R A D}$ é o calor transferido à câmara por irradiação $(W), h_{\text {TRAFO }}$ é o coeficiente de transferência de calor por convecção $\left(W /\left(m^{2} K\right)\right), A_{\text {TRAFO }}$ é a área externa exposta do transformador $\left(m^{2}\right), T_{\text {TRAFO }}$ é a temperatura do transformador $\left({ }^{\circ} \mathrm{C}\right), \mathrm{T}_{A R}$ é a temperatura média do ar no interior da câmara $\left({ }^{\circ} \mathrm{C}\right), \sigma$ é a constante de Stefan Boltzman $\left(5,67 \times 10^{-8} \mathrm{~W} /\left(\mathrm{m}^{2} \mathrm{~K}^{4}\right)\right)$, $\varepsilon_{\text {TRAFO }}$ é a emissividade média da superfície do transformador, $A_{C}$ é a área de superfície das paredes e teto da câmara, e $\varepsilon_{C}$ é a emissividade média da superfície das paredes e teto da câmara.

A Eq. (12) é utilizada para a determinação da temperatura do transformador:

$$
\begin{array}{r}
C_{\text {TRAFO }} \frac{d T_{T R A F O}}{d t}=P_{F E}+s^{2} P_{c u}-h_{\text {TRAFO }} \times \\
A_{\text {TRAFO }}\left(T_{\text {TRAFO }}-T_{A R}\right)-\frac{\sigma A_{T R A F O}\left(A_{T R A F}^{4}-T_{A R}^{4}\right)}{R_{T}}
\end{array}
$$

sendo que: $P_{F E}$ é a perda no núcleo de ferro do transformador para a tensão nominal $(W)$, s é o carregamento do transformador em unidade de sua potência nominal, $P_{c u}$ é a perda no cobre do transformador para a plena carga $(W)$.

Uma rede neural difusa é utilizada por Rigatos et al. (2012) para modelar a condição térmica do transformador de potência em operação sem falhas (a condição térmica está associada a uma variável de temperatura conhecida como temperatura do ponto quente). A saída da rede neural difusa é comparada com as medições do transformador de potência, e os resíduos obtidos sofrem processamento estatístico de acordo com um algoritmo de detecção e isolamento de falhas. Neste sentido, para a obtenção de um modelo analítico do comportamento térmico do transformador de potência primeiramente foi calculado, conforme Eq. (13), a cada passo de tempo, o aumento da temperatura do óleo no transformador a partir da corrente de carga naquele instante, dado por:

$$
\Delta \Theta_{T O, U}=\Delta \Theta_{T O, R}\left[\frac{I_{L}^{2} R+1}{R+1}\right]^{q}
$$

sendo que: $\Delta \Theta_{\mathrm{TO}, U}$ é o aumento da temperatura máxima do óleo $\left({ }^{\circ} \mathrm{C}\right), \Delta \Theta_{T O, R}$ é o aumento nominal da temperatura máxima do óleo acima da temperatura ambiente $\left({ }^{\circ} \mathrm{C}\right), I_{L}$ é a corrente da carga normalizada para corrente nominal (p.u.), $q$ é o expoente empiricamente derivado para explicar aproximadamente os efeitos da mudança de resistência com a mudança na carga, e $R$ é a relação entre perda de carga nominal e perda sem carga na posição de derivação aplicável.

Em seguida, foi calculado na Eq. (14) o incremento na temperatura do óleo a partir da variação desta e sua relação com a temperatura ambiente::

$$
\tau_{T O} \frac{d \Theta_{T O}}{d t}=\left[\Delta \Theta_{T O, U}+\Theta_{A}\right]-\Theta_{T O}
$$

sendo que: $\Theta_{T O}$ é a temperatura máxima do óleo $\left({ }^{\circ} \mathrm{C}\right)$, $\tau_{T O}$ é a constante de tempo de aumento de óleo superior $\left({ }^{\circ} \mathrm{C}\right)$, e $\Theta_{A}$ é a temperatura ambiente $\left({ }^{\circ} \mathrm{C}\right)$.

Na sequência, na Eq. (15) foi calculado o aumento final da Temperatura do Ponto Quente (TPQ) dado por:

$$
\Delta \Theta_{H S, U}=\Delta \Theta_{H S, R} I_{L}^{2 \beta}
$$

sendo que: $\Delta \Theta_{H S, U}$ é o último aumento da TPQ do transformador sobre o óleo superior para uma determinada corrente de carga $\left({ }^{\circ} \mathrm{C}\right), \Delta \Theta_{H S, R}$ é o aumento nominal da TPQ sobre o óleo superior para corrente de carga nominal $\left({ }^{\circ} \mathrm{C}\right)$, e $\beta$ é um expoente empiricamente derivado dependente do método de resfriamento.

Ainda, na Eq. (16) foi calculado o incremento da TPQ:

$$
\tau_{H S}\left\{\frac{d \Delta \Theta_{H S}}{d t}\right\}=\Delta \Theta_{H S, U}-\Delta \Theta_{H S}
$$

sendo que: $\Theta_{H S, U}$ é a temperatura do enrolamento do ponto quente $\left({ }^{\circ} \mathrm{C}\right)$.

Finalmente, na Eq. (17) foi adicionada a temperatura do óleo para obter a TPQ, dada por:

$$
\Theta_{H S}=\Theta_{T O}-\Delta \Theta_{H S} .
$$

Sandraz et al. (2013) propõem um novo modelo térmico para transformadores subterrâneos que utiliza parte do modelo original de Whirlow and Lockie (1968) levando em consideração algumas características importantes da instalação do transformador, que são: formas retangulares, bobina e núcleo, orientação da convecção para ar (fluxos de calor vertical, horizontal para cima e horizontal descendente) e regime de fluxo turbulento ou laminar. O modelo é apresentado na Fig. 3, e foi validado utilizando três transformadores com dimensões distintas instalados em diferentes câmaras, com medições no local.

A analogia térmica elétrica permite descrever o fluxo 


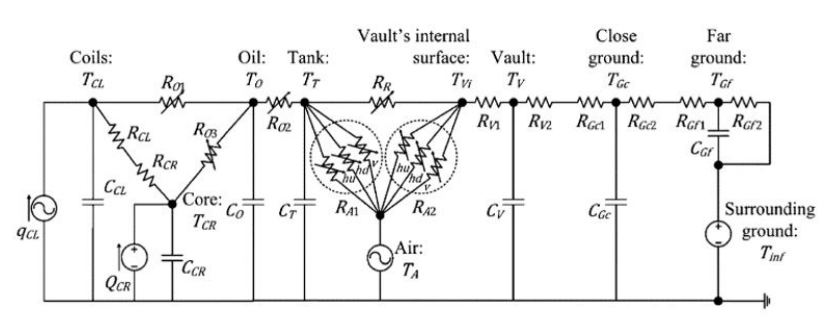

Figura 3: Representação da transferências de calor.

de calor com um circuito de parâmetro agrupado da mesma maneira que flui uma corrente elétrica. Existem três principais mecanismos para o transporte: a condução entre os sólidos, a convecção entre um sólido e o seu fluido circundante, e a radiação entre dois sólidos através de um fluido. O sistema apresentado na Fig. 3 é descrito pela equação diferencial-algébrica, em Eq. (18), dada por::

$$
M(T) \frac{d T(t)}{d t}=A(T) T(t)+B(T) u(t)
$$

sendo que, na Eq. (19):

$$
T(t)=\left[\begin{array}{llllllll}
T_{C L} & T_{C R} & T_{O} & T_{T} & T_{V i} & T_{V} & T_{G c} & T_{G f}
\end{array}\right]
$$

é o vetor temperatura $\left({ }^{\circ} \mathrm{C}\right)$, e na Eq. (20):

$$
u(t)=\left[\begin{array}{llll}
q_{C L}(t) & T_{A}(t) & T_{A}(t) & T_{i n f}
\end{array}\right]^{T}
$$

é o vetor contendo os fluxos de calor $\left(W / m^{2}\right), M(T)$ é a matriz de massa $(\mathrm{kg}), A(T)$ é a matriz de dinâmica, e $\mathrm{B}(\mathrm{T})$ é uma matriz de entradas.

Em Cui et al. (2016) é proposto um Modelo Térmico Dependente da Umidade (MTDU) para estimar a TPQ do transformador, uma vez que a TPQ é reconhecida como um dos principais fatores que afetam a sua expectativa de vida. Neste modelo, a resistência térmica não linear é formulada considerando tanto o óleo, como a celulose, mais especialmente o efeito da concentração de umidade e da temperatura na resistência térmica da celulose do transformador.

O MTDU proposto é verificado usando dados de medições da umidade em óleo, temperatura e perfis de carga, que foram coletados de dois transformadores de potência em serviço, isto é, um transformador de óleo vegetal e um transformador com óleo mineral. As comparações entre o MTDU proposto e um número de modelos térmicos existentes foram realizadas com base nas métricas de adequação e precisão. Um aumento na corrente de enrolamento de um transformador resultará em um aumento de temperatura no óleo e no enrolamento. $O$ aumento da temperatura do óleo superior pode ser calculado como uma resposta exponencial do aumento inicial da temperatura do óleo superior $\Delta \Theta_{o i l, I}$ para o aumento final da temperatura $\Delta \Theta_{o i l, U}$ conforme as Eqs. (21) e (22):

$$
\begin{gathered}
\tau_{o i l, R}=\frac{d \Delta \Theta_{o i l}}{d t}=\Delta \Theta_{o i l, U}-\Delta \Theta_{o i l, I} \\
\Delta \Theta_{o i l, U}=\Delta \Theta_{o i l, R}\left(\frac{1+R . K^{2}}{1+R}\right)
\end{gathered}
$$

sendo que: $\tau_{o i l, R}$ é a constante de tempo do óleo, $\Delta \Theta_{o i l, R}$ é o aumento da temperatura do óleo nominal acima da temperatura ambiente, $R$ é a relação entre $q C u$ (perda de carga do transformador) e $q F e$ (perda do transformador durante teste sem carga), $K$ é a relação entre corrente de carga e corrente de enrolamento nominal, $\Delta \Theta_{o i l, U}$ é o aumento final da temperatura do óleo superior ao ambiente, $\Delta \Theta_{o i l, I}$ é o aumento inicial da temperatura do topo do óleo acima da temperatura ambiente. Por fim, na Fig. 4 é apresentado esse modelo dinâmico da temperatura superior do óleo.

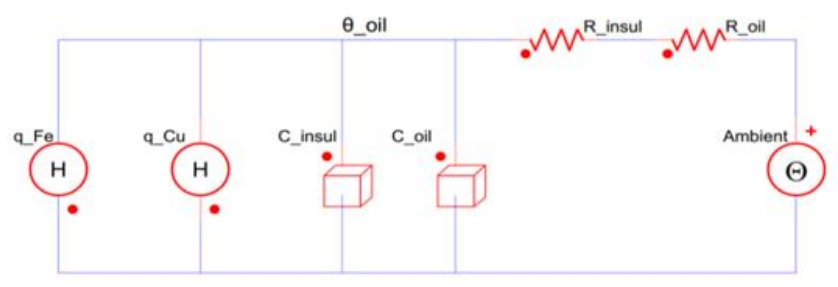

Figura 4: Modelo dinâmico de temperatura.

A partir da revisão bibliográfica realizada nesta pesquisa foi definida pela utilização do modelo de Whirlow and Lockie (1968), pois além dele ser o precursor dos modelos térmicos para o ambiente subterrâneo, outros modelos foram derivados a partir dele, tais como, os modelos de Sandraz et al. (2013) e Djamali et al. (2018). A modelagem matemática proposta neste artigo utiliza parte das equações originais do modelo de Whirlow and Lockie (1968). Adaptações ao modelo são realizadas para trabalhar com a carga do transformador e os dados da temperatura do topo do óleo. Os dados para a validação do modelo foram obtidos de um sistema de monitoramento de uma subestação subterrânea de energia de uma concessionária de energia elétrica do sul do Brasil.

\section{Sistema de Distribuição de Energia}

O sistema de distribuição reticulado de energia utilizado como estudo de caso, neste artigo, está localizado no centro da cidade de Porto Alegre, sendo alimentado com tensões primárias de $13,8 \mathrm{kV}$ e tensões secundárias de 127/220 V. Ele é formado por 160 subestações subterrâneas, com características semelhantes e compostas essencialmente por transformadores de $500 \mathrm{kVA}$, submersíveis (IP68), pertencendo a Companhia Estadual de Energia Elétrica do Rio Grande do Sul (CEEE/RS). 
Um sistema de monitoramento, não invasivo, das condições de operação destas subestações foi desenvolvido utilizando comunicação PLC (Power Line Communication).

Na Fig. 5 as grandezas que são monitoradas podem ser observadas graficamente sendo divididas entre grandezas analógicas e digitais. As grandezas analógicas monitoradas são a corrente no primário, a tensão e a corrente no secundário, e a temperatura da carcaça do transformador, além da temperatura interna da subestação e a temperatura ambiente. As demais grandezas são do tipo digital, como por exemplo, o estado (ligado ou desligado) das bombas, ventiladores e indicativos luminosos do transformador.

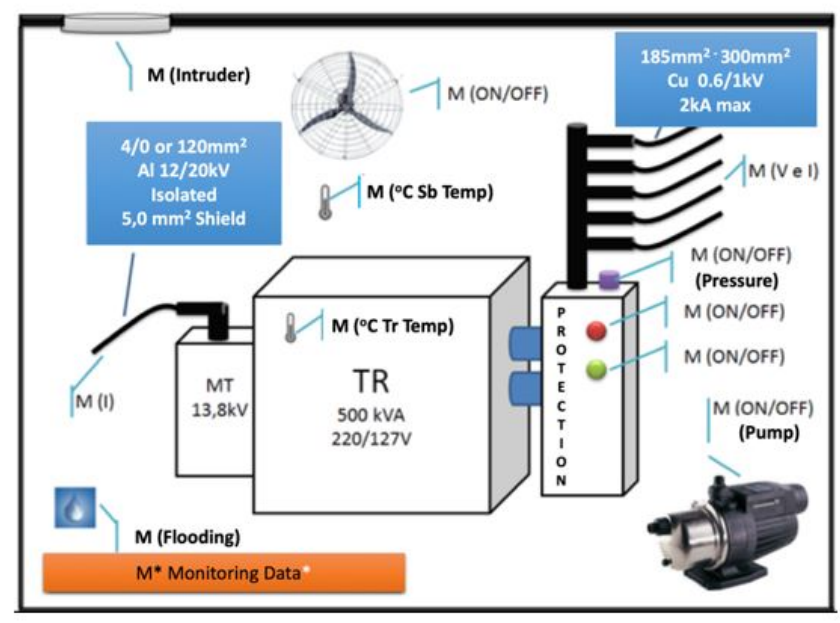

Figura 5: Grandezas monitoradas pelo sistema.

Os maiores riscos neste tipo de sistema são as inundações, superaquecimento, falha no sistema de proteção, roubos e alterações na pressão do sistema de proteção. $O$ sistema deve também ser apto a monitorar o alagamento da subestação e a entrada de intrusos. As medições são realizadas a cada 10 segundos, assim permitindo a construção de um banco de dados, que associado aos problemas encontrados nos últimos dois anos, possibilitam que um conjunto de pesquisas possam ser desenvolvidas. Na Fig. 6 é apresentada uma fotografia da subestação que é utilizada neste trabalho como referência, e na Tabela 1 são mostrados os dados do transformador.

Para que seja possível continuar com o processo de automação da subestação, são necessários que os modelos matemáticos das grandezas monitoradas sejam corretamente desenvolvidos e validados. A partir disto, este sistema que compõe essa subestação poderá representar uma aplicação onde será possível, por exemplo, a detecção de faltas no sistema subterrâneo reticulado e ainda prever seu comportamento nas mais diversas condições de operação.

Neste contexto, nesse artigo é apresentada a modelagem matemática do comportamento térmico dessa subestação considerando a influência de duas tempera-

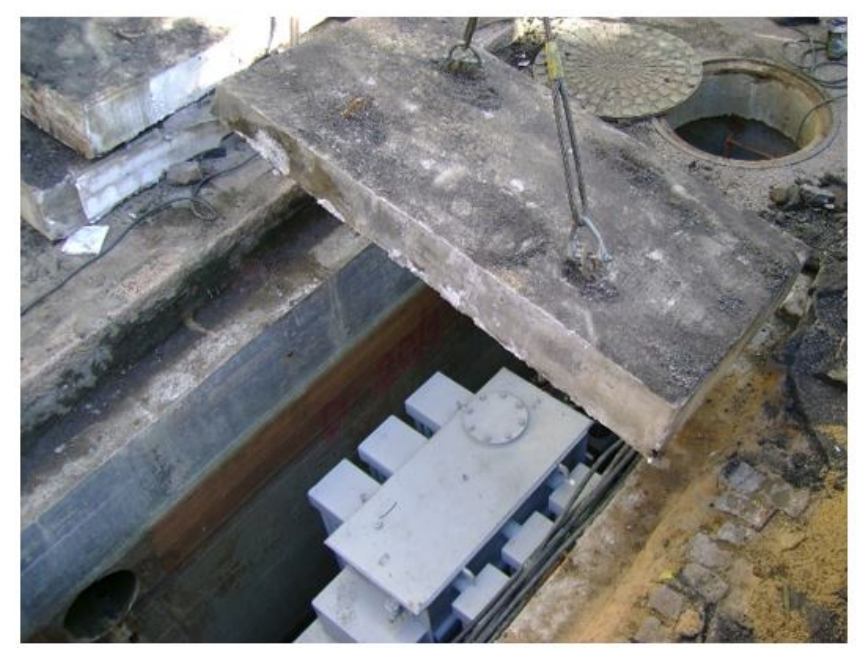

Figura 6: Subestação Subterrânea.

Tabela 1: Dados do Transformador Trifásico

\begin{tabular}{lc} 
Norma Aplicada & ABNT NRB 5356 \\
Tipo & TCY500 \\
Material Primário & Cobre \\
Material Secundário & Cobre \\
Potência do Transformador & $500 \mathrm{kVA}$ \\
Frequência & $60 \mathrm{~Hz}$ \\
Temperatura de Referência & $75^{\circ} \mathrm{C}$ \\
Classe de Tensão Primário & $15 \mathrm{kV}$ \\
NBI AT & $110 \mathrm{kV}$ \\
Tensão no Primário & $13,8 \mathrm{kV}$ \\
Corrente Nominal no Primário & $20,9 \mathrm{~A}$ \\
Classe de Tensão Secundário & $1,2 \mathrm{kV}$ \\
NBI BT & $30 \mathrm{kV}$ \\
Tensão no Secundário & $220 \mathrm{~V}$ \\
Corrente Nominal no Secundário & $1312,16 \mathrm{~A}$ \\
\hline
\end{tabular}

turas: a temperatura ambiente de Porto Alegre $\left(T_{A}\right)$ e a temperatura da carcaça do transformador subterrâneo $\left(T_{T}\right)$. Foram considerados neste estudo o período de 72 horas correspondentes aos dias 9, 10 e 11 de julho e 6,7 e 8 de dezembro de 2018 , inverno e verão respectivamente.

\section{Modelo Térmico}

A modelagem matemática realizada neste artigo teve como base o trabalho desenvolvido por Whirlow and Lockie (1968), na qual adaptações ao modelo foram realizadas para adequar o mesmo a realidade do estudo de caso utilizado nesta pesquisa. Conforme Sandraz et al. (2013), a analogia termoelétrica permite descrever o fluxo de calor através de um circuito de parâmetros concentrados, sendo a corrente elétrica que flui nesse circuito o fluxo. Existem três mecanismos principais para a transferência de calor: condução entre dois sólidos, convecção entre um sólido e seu fluido circundante, e radiação entre dois sólidos através de um fluido. A condução pode ser modelada por uma resis- 
tência térmica constante, mas a convecção e a radiação são fenômenos não lineares.

Neste sentido, na Fig. 7 é apresentada a proposta do circuito elétrico equivalente de um modelo térmico reduzido, baseado no modelo apresentado em Whirlow and Lockie (1968) e adaptado por Sandraz et al. (2013). O modelo é composto por resistências e capacitâncias térmicas referentes a cada temperatura analisada no estudo de caso. Com base no circuito elétrico proposto é possível realizar o equacionamento matemático do modelo.

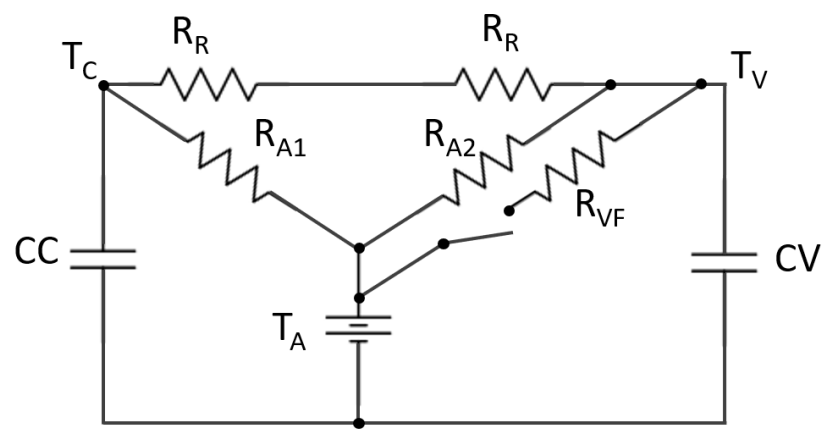

Figura 7: Circuito equivalente do modelo proposto.

A equação que governa o seu comportamento é apresentada na Eq. (23), a qual resulta na temperatura da parede interior da subestação subterrânea, dada por:

$$
T_{V}=I_{R V F} \frac{h_{R} A_{C O} T_{T}+h_{A 2} A_{V} T_{A}+\frac{h_{R T}}{r_{V}}}{h_{R} A_{C O}+h_{A 2} A_{V}+\frac{1}{r_{V}}}
$$

sendo que: $I_{R V F}$ é o índice de resfriamento de ventilação forçada, $h_{R}$ é o coeficiente de radiação $\left(\mathrm{W} / \mathrm{m}^{2} .{ }^{\circ} \mathrm{C}\right), A_{C O}$ é a área do óleo $\left(\mathrm{m}^{2}\right), \mathrm{T}_{T}$ é a temperatura da carcaça do transformador $\left({ }^{\circ} \mathrm{C}\right), h_{A 2}$ é o coeficiente de convecção do $\operatorname{ar}\left(W / m^{2} \cdot{ }^{\circ} \mathrm{C}\right), A_{V}$ é a área da câmara subterrânea $\left(m^{2}\right)$, $\mathrm{T}_{A}$ é a temperatura ambiente de Porto Alegre $\left({ }^{\circ} \mathrm{C}\right), h_{R T}$ é o coeficiente de radiação do transformador na câmara subterrânea $\left({ }^{\circ} \mathrm{C}\right)$, e $r_{V}$ é a resistência térmica da câmara $\left({ }^{\circ} \mathrm{C} / W\right)$.

O coeficiente de radiação $\left(h_{R}\right)$ pode ser determinado através da Eq. (24), a qual considera a temperatura ambiente da cidade de Porto Alegre e a temperatura da carcaça do transformador, dada por:

$$
h_{R}=0,00882\left[\left(\frac{T_{A}}{100}\right)^{2}+\left(\frac{T_{T}}{100}\right)^{2}\right]\left(\frac{T_{A}+T_{T}}{100}\right) .
$$

O coeficiente de convecção $\left(h_{A 2}\right)$ referente a área, pode ser determinado pela Eq. (25):

$$
h_{A 2}=5,54\left(\frac{T-T_{A}}{T_{A}}\right)^{1 / 4}
$$

sendo que: T é a temperatura de referência $\left({ }^{\circ} \mathrm{C} / W\right)$.

0 coeficiente de radiação do transformador $\left(h_{R T}\right)$ na câmara subterrânea pode ser determinado pela Eq. (26):

$$
h_{R T}=R_{A C} T_{T}
$$

sendo que: $R_{A C}=\frac{1}{A_{V} * 0,035}$ é a resistividade térmica do ar interno da câmara, e 0, 035 é a condutividade térmica do ar.

\section{Resultados das Simulações}

Os dados da temperatura da carcaça do transformador $\left(T_{T}\right)$ são provenientes do sistema de monitoramento das subestações de energia elétrica. Esse sistema foi desenvolvido em parceria com a CEEE/RS, portanto os dados são reais e refletem exatamente o comportamento de uma subestação nos períodos indicados.

Na subestação considerada, a câmara em que o transformador está instalado tem as seguintes dimensões: 1,7 metros de altura, 8 metros de comprimento e 3 metros de largura. Além disso, a subestação possui um sistema de arrefecimento, onde a entrada de ar é por grades de ventilação e a saída de ar é forçada em um duto; e um sistema de bombeamento, onde o esgotamento de água ocorre a partir de um alagamento, sendo realizado por uma bomba hidráulica.

A temperatura ambiente de Porto Alegre $\left(T_{A}\right)$ pode ser encontrada a partir do banco de dados do Weather Spark, que fornece relatórios detalhados do clima típico de 150.165 localidades distribuídas por todo o mundo. Dois ciclos de temperatura são utilizados na análise, um referente ao período de inverno e outro referente ao período do verão. Em ambos os casos, o ciclo de temperatura ambiente utilizado corresponde a 72 horas, conforme pode ser observado na Fig. 8.

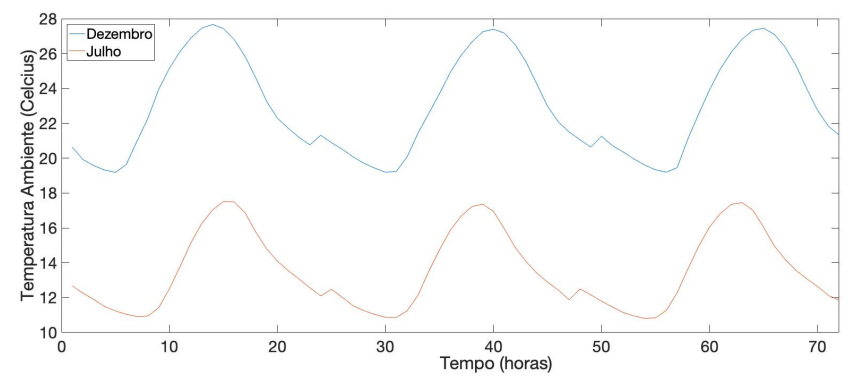

Figura 8: Ciclos de temperatura ambiente.

Na Fig. 9, pode-se observar a eficiência do transformador utilizado na análise, para os dados dos meses de julho e dezembro obtidos a partir do sistema de monitoramento para o período de 72 horas.

Na Fig. 10 é apresentada a potência de operação do transformador durante o período analisado. Cabe destacar, que durante a análise, essa potência de operação e consequentemente a eficiência do transformador no 


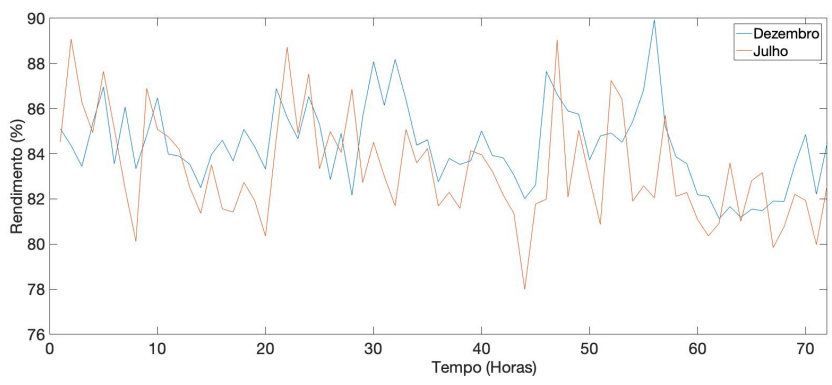

Figura 9: Eficiência do transformador de potência.

mês de julho (apresentado na Fig. 9) foi menor quando comparado ao mês de dezembro. Na Fig. 11 pode-se observar a temperatura da carcaça do transformador.

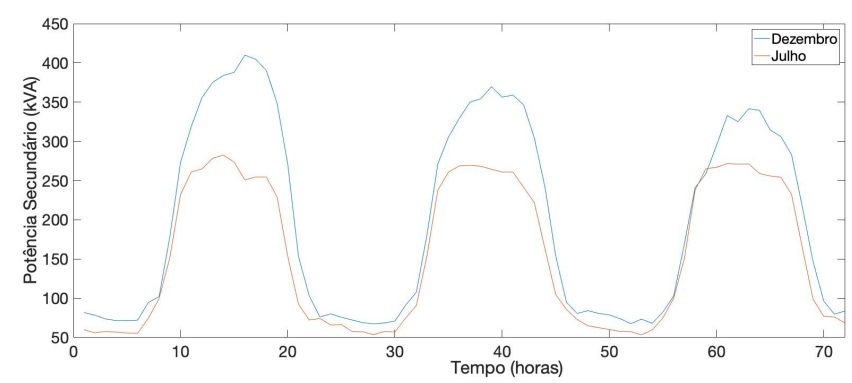

Figura 10: Potência no secundário do transformador.

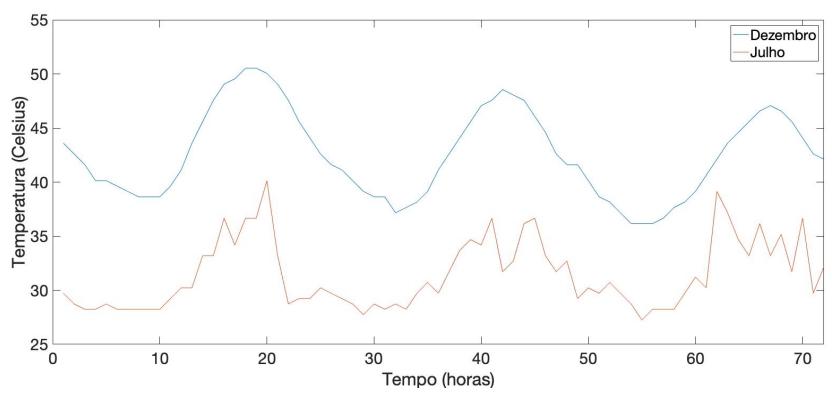

Figura 11: Ciclos de temperaturas da carcaça do transformador.

Então, a partir dos dados apresentados, foram realizadas simulações com o modelo proposto, e os resultados destas simulações foram comparados com os dados reais das subestações, objetivando desta forma realizar a validação do modelo proposto. Os resultados do processo de validação do modelo são apresentados na Fig. 12 para o mês de julho, e na Fig. 13 para o mês de dezembro.

Analisando as Figs. 12 e 13 é possível observar que o modelo proposto converge para as temperaturas medidas. Visando verificar a sua acurácia foi calculado o erro

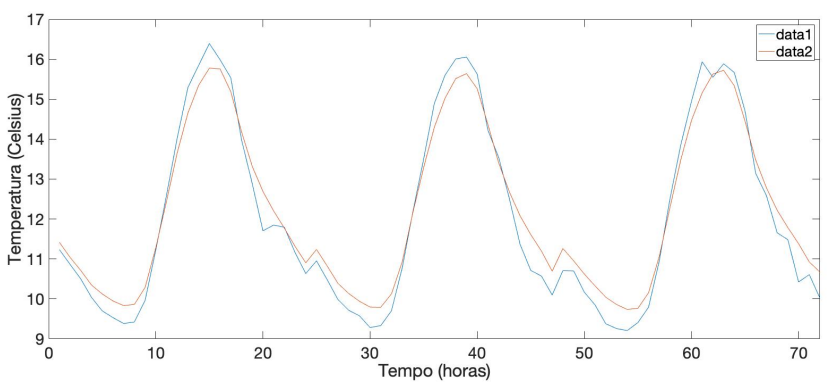

Figura 12: Temperatura interna da subestação para o mês de julho.

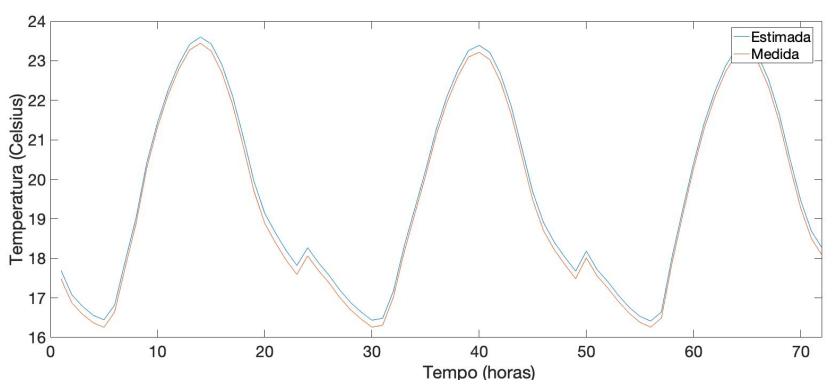

Figura 13: Temperatura interna da subestação para o mês de dezembro.

a partir da Eq. (27) que reflete a diferença em termos percentuais entre o valor da temperatura medida pelo sistema de monitoramento, e o valor da temperatura calculada pelo modelo, ou seja:

$$
\text { erro }_{\text {rel }}=\frac{\left|T_{M}\right|-\left|T_{C}\right|}{\left|T_{M}\right|} \times 100 \%
$$

onde: $T_{M}$ é a temperatura medida na subestação subterrânea, $T_{C}$ é a temperatura calculada pelo modelo.

$\mathrm{O}$ erro resultante entre a temperatura calculada pelo modelo proposto e a temperatura medida na subestação foi de 3,09 \% para o mês de junho e de $0,88 \%$ para o mês de dezembro. Ambos percentuais, inferiores a 5 $\%$, estão dentro da faixa de erro que outros modelos térmicos obtiveram em trabalhos correlatos, tais como em Ryadi and Tanguy (2018), Mharakurwa et al. (2018) e Zhou et al. (2020).

\section{Conclusões}

Os transformadores são fundamentais para a operação do SEP, além disso, considerando a rápida evolução dos sistemas de instrumentação proporcionado pelo avanço das redes inteligentes, modelos matemáticos acurados e devidamente validados são essenciais para a determinação do comportamento térmico de subestações subterrâneas de energia. $O$ presente artigo apresentou a modelagem matemática do comportamento térmico de uma subestação subterrânea considerando a influên- 
cia de duas temperaturas, a ambiente da cidade e a temperatura da carcaça do transformador. Foram considerados nesta pesquisa o mês de julho e dezembro de 2018 , inverno e verão respectivamente. Por fim, a partir dos resultados das simulações é possível observar que o modelo proposto descreveu o comportamento térmico da subestação com erros inferiores a 3,1\%.

\section{Agradecimentos}

A Companhia Estadual de Distribuição de Energia Elétrica (CEEE-D/RS) e a Agência Nacional de Energia Elétrica (ANEEL) pelo apoio financeiro.

\section{Referências}

Cui, Y., Ma, H., Saha, T., Ekanayake, C. and Martin, D. (2016). Moisture-dependent thermal modelling of power transformer, IEEE Transactions on Power Delivery 31(5): 2140-2150. http://dx.doi.org/10.1109/ TPWRD . 2016.2569123.

Djamali, M. and Tenbohlen, S. (2017). Hundred years of experience in the dynamic thermal modelling of power transformers. http://dx.doi.org/10.1049/ iet-gtd.2016.1321.

Djamali, M., Tenbohlen, S., Junge, E. and Konermann, M. (2018). Real-time evaluation of the dynamic loading capability of indoor distribution transformers, IEEE Transactions on Power Delivery 33(3): 1134-1142. http://dx.doi.org/10.1109/TPWRD.2017.2728820.

Gouda, O. E., El Dein, A. Z. and Moukhtar, I. (2013). Turn-to-earth fault modelling of power transformer based on symmetrical components, IET Generation, Transmission Distribution 7(7): 709-716. http: //dx.doi.org/10.1049/iet-gtd.2012.0724.

Gouvea, M. R., Robba, E. J., Belvedere, E. C., Moreira, J. R. S. and Brunheroto, P. A. (2004). Thermal simulation for distribution transformers in underground vaults, 2004 IEEE/PES Transmision and Distribution Conference and Exposition: Latin America (IEEE Cat. No. 04EX956), pp. 987-991. http://dx.doi.org/ 10.1109/TDC. 2004.1432517.

Lockie, A. M. and Whirlow, D. K. (1968). Thermal performance of distribution transformers in underground vaults: I - the problem, IEEE Transactions on Power Apparatus and Systems PAS-87(9): 1741-1745. http://dx.doi.org/10.1109/TPAS.1968.291978.
Mharakurwa, E. T., Nyakoe, G. N. and Akumu, A. O. (2018). Thermal modeling of power transformers with unbalanced loading, 2018 IEEE PES/IAS PowerAfrica, pp. 126-130. https://doi.org/10.1109/ PowerAfrica.2018.8521168.

Rigatos, G., Siano, P. and Piccolo, A. (2012). Incipient fault detection for electric power transformers using neural modeling and the local statistical approach to fault diagnosis, 2012 IEEE Sensors Applications Symposium Proceedings, pp. 1-6. http://dx.doi.org/ 10.1109/SAS.2012.6166269.

Ryadi, M. and Tanguy, A. (2018). Field validated dynamic thermal model for power transformer insulation system assessment, 2018 IEEE Electrical Insulation Conference (EIC), pp. 101-105. https://doi.org/10.1109/ EIC. 2018.8481065.

Sandraz, J. P. A., de León, F. and Cultrera, J. (2013). Validated transient heat-transfer model for underground transformer in rectangular vault, IEEE Transactions on Power Delivery 28(3): 1770-1778. http: //dx.doi.org/10.1109/TPWRD. 2013.2260183.

Shirvani, A., Malekian, K., Schmidt, U. and Schufft, W. (2010). A new power transformer model over wide frequency rang for emtp, 45th International Universities Power Engineering Conference UPEC2010, pp. 1-6.

Theis, N. (2008). Power Systems Modelling and Fault Analysis - Theory and Practice, 1 edn, Elsevier Ltda.

Whirlow, D. K. and Lockie, A. M. (1968). Thermal performance of distribution transformers in underground vaults: Ii - mathematical model, IEEE Transactions on Power Apparatus and Systems PAS87(9): 1745-1754. http://dx.doi.org/10.1109/TPAS. 1968.291979.

Zhou, B., Xu, X., Or, S. W., Li, C., Wu, Q., Zhang, C. and $\mathrm{Li}, \mathrm{W}$. (2020). Thermodynamic modelling of buried transformer substations for dynamic loading capability assessment considering underground heat accumulative effect, International Journal of Electrical Power \& Energy Systems 121: 106153. http://dx.doi. org/10.1016/j.ijepes.2020.106153.

Zhou, D., Wang, Z., Jarman, P. and Li, C. (2014). Data requisites for transformer statistical lifetime modelling-part ii: Combination of random and aging-related failures, IEEE Transactions on Power Delivery 29(1): 154-160. http://dx.doi.org/10.1109/ TPWRD . 2013.2270116. 